\title{
High-redshift quasars and blazars and their relation to high-redshift galaxies
}

\author{
Areg Mickaelian, Hayk Abrahamyan and Gurgen Paronyan \\ Byurakan Astrophysical Observatory (BAO), Byurakan 0213, Aragatzotn Province, Armenia \\ email: aregmick@yahoo.com
}

\begin{abstract}
High-redshift quasars and blazars are among the most powerful sources in the Universe and among the highest luminosity sources, as in optical, so as in X-ray, UV, IR and radio range. We have created a full sample of blazars and high-redshift quasars $(z \geqslant 2.15)$. The evolutionary relation of high-redshift quasars and blazars to high-redshift galaxies is discussed using the luminosity evolution.
\end{abstract}

Keywords. galaxies: active, quasars: general, BL Lacertae objects: general

Because of their high luminosity, powerful quasars and blazars are being observed at highest redshifts, as in optical, so as in X-ray, UV, IR and radio ranges. However, their evolutionary relation to high-redshift galaxies is not yet clear. Have they formed at the same epoch or there is an evolutionary transition between these objects? There are many similarities indicating such connections, such as the high luminosity, radio and $\mathrm{X}_{-}$ ray emissions, jets, etc. We have investigated this subject on the basis of observational data to find relations and possible evolutionary links. We have created a full sample of blazars and high-redshift quasars $(\mathrm{z} \geqslant 2.15)$ based on their lists from Véron-Cetty \& Véron (2010), LQAC2 (Souchay et al. 2012) and Roma-BZCAT (Massaro et al. 2015) catalogues and newly discovered objects from SDSS and other surveys (DR10Q; Pâris et al. 2014). Due to SDSS III BOSS DR10 Quasar Catalog, the total number of QSOs is 334,210 , including $134,271(40.2 \%)$ with $\mathrm{z} \geqslant 2.15$. Most of these objects were discovered in SDSS, as objects with $z \geqslant 2.15$ in Véron-Cetty \& Véron (2010) are only $15.6 \%$.

The inhomogeneity of data (optical photometry and fluxes at other wavelength ranges, variability, etc.) does not allow correct statistics of the physical parameters. Therefore we started from the creation of a homogeneous sample based on all parameters. We selected only bright $(\mathrm{V} \leqslant 19)$ objects also to have multiwavelength data from gamma-ray, $\mathrm{X}$-ray, UV, IR and radio all-sky or large area surveys and we were left with a sample of 14,135 objects. Most of these objects show resolved images in SDSS $g, r$, and $i$, as well as in DSS2 $B, R$ and $I$. Many of QSOs may also turn to be blazars if studied in detail.

The luminosity function (LF) for this sample was built and studied. LF for the full sample of 134,271 high- $z$ objects shows a secondary peak at $\mathrm{M}_{V} \sim 18.5$, probably connected with the existance of stellar population around these objects and measured total luminosity compared to fainter objects, for which only the luminosity of the core is given. The evolutionary relation of high $-z$ quasars and blazars to high $-z$ galaxies is discussed.

\section{References}

Massaro, E., Maselli, A., Leto, C., et al. 2015, Apళ6SS, 357, 75

Pâris, I., Petitjean, P., Aubourg, É., et al. 2014, A\&A, 563, A54

Souchay, J., Andrei, A. H., Barache, C., et al. 2012, A\&A, 537, A99

Véron-Cetty, M. P., \& Véron, P. 2010, A\&̈A, 518, A10 